\title{
Learning how to learn: Can experiencing the outcome of different encoding strategies enhance subsequent encoding?
}

\author{
Elizabeth LIGON BJORK \\ University of California, Los Angeles, California \\ Patricia Ann deWinstanley \\ Oberlin College, Oberlin, Ohio \\ AND \\ Benjamin C. Storm \\ University of California, Los Angeles, California
}

\begin{abstract}
Research on how individuals monitor their level of comprehension during study paint a picture of learners as insensitive to many of the factors or conditions of learning that can enhance long-term retention and transfer. In the present article, we discuss research examining the sensitivity, or lack thereof, of learners to one such factor: generation. More specifically, we discuss research addressing the question of learners' sensitivity to the memorial benefits of generation and whether - if given the opportunity to experience this benefit in their own recall performance - they might then go on to develop enhanced encoding strategies in the processing of new to-be-learned information.
\end{abstract}

How individuals monitor their level of comprehension during study is a question of growing interest to researchers of metacognitive processes (see, e.g., Benjamin, Bjork, \& Schwartz, 1997; Bjork, 1999; Dunlosky \& Nelson, 1994; Koriat, 1997, 1998). Indeed, some argue (e.g., Bjork, 1999; Jacoby, Bjork, \& Kelley, 1994) that the readings learners take on their level of comprehension while studying can be as important as their actual comprehension, because, in part, such readings influence their decisions on how to allocate learning resources. On the basis of such readings, for example, students may decide to review one chapter rather than another, or to spend more time studying one set of materials versus another in preparation for an examination.

Learners, however, can be far from accurate in taking such readings and can be subject to illusions of comprehension (see, e.g., Bjork, 1999; Jacoby et al., 1994). They can, for example, be led to think that their level of comprehension or skill is greater than it actually is, owing to conditions of learning (such as massed practice) that enhance or support performance during study or training but actually impair long-term retention and/or transfer (Bjork, 1999; Simon \& Bjork, 2001). Koriat (1997) has stated this slightly differently, arguing that learners can suffer from illusions of competence because they are relatively insensitive to factors in the learning environment (such as repeated presentations) that can enhance performance on later retention tests, while being overly sensitive to factors (such as the perceived association between cues and targets when both are present during study) that do not necessarily enhance performance on later retention tests. In the present article, we describe research exploring the sensitivity, or lack thereof, of learners to the memorial benefits of one such factor: generation.

In the sections below, we first define and illustrate the generation effect or advantage, describe two accounts of it that are consistent with a wide body of relevant findings, and then discuss our more recent research addressing the general issue of learners' sensitivity to the memorial benefits of generation and whether - if made sensitive to this benefit - they might then adopt more effective encoding strategies in the processing of new information.

\section{Generation As a Condition of Learning}

When learners take an active part in generating the information they are learning, as opposed to having it provided to them, they tend to remember it better. If, for example, learners generate the word banana from a word fragment (e.g., $b-n-n-$ ), as opposed to being given the intact word to read, they will recall it better on a later test. Or, if required to generate the exemplar banana to a category-plus-letter-stem cue (e.g., Fruit-ba versus

E. L. Bjork, elbjork@psych.ucla.edu 
being given the intact pair to study, they will recall banana better in response to the cue Fruit on a later test.

This memorial benefit of generation (see, e.g., Jacoby, 1978; Slamecka \& Graf, 1978) has proved to be both robust and to extend to a variety of learning materials, including lists of words, trivia questions (e.g., deWinstanley, 1995), and mathematical problems (e.g., McNamara \& Healy, 1995a, 1995b; Pesta, Sanders, \& Murphy, 1999). Under certain conditions, however, the generation effect can be diminished or even eliminated. McNamara and Healy (1995a, 1995b), for example, found that generation advantages do not occur for arithmetic problems unless retrieval strategies that reinstate procedures employed during study are evoked again at the time of test.

Similarly, deWinstanley, Bjork, and Bjork (1996) demonstrated that even when participants learn the same materials, generation advantages may or may not occur, depending on the match between the information strengthened during the generation task and the type of information required for optimal performance on a later test. More specifically, the conditions of learning were manipulated to force the processing of different types of information in order to generate targets for the same set of cue-target pairs. In one condition, the pairs were blocked into categories, leading participants to focus on target-target relational information (to which free recall tests are assumed to be most sensitive) rather than cue-target relational information (to which cued recall tests are assumed to be most sensitive) in order to perform the generation task. On subsequent tests, participants showed a generation advantage when given a free recall test, but not when given a cued recall test. In the other condition, pairs were not blocked by category, essentially eliminating target-target relational processing as a basis for generating targets and forcing participants to rely on cue-target relational information instead. On subsequent tests, these participants showed a generation advantage on a cued recall test, but not on a free recall test. In other words, a striking reversal was observed in the relative levels of free and cued recall for targets that had been generated versus read, depending on the type of information that participants were forced to use to generate the targets during learning.

Whether generation effects occur can also be influenced by the encoding instruction given to learners. Begg, Vinski, Frankovich, and Holgate (1991), for example, showed that generation advantages could be eliminated by giving participants effective strategies, such as imagery, to use when encoding to-be-read items. Similarly, deWinstanley and Bjork (1997) eliminated a previously observed generation advantage by giving participants explicit instructions concerning the type of retention test to expect and how to process information optimally in anticipation of such a test.

\section{Accounts of the Generation Advantage}

These types of findings delineating the circumstances under which generation advantages do and do not occur are consistent with two explanations of generation effects: the procedural account and the transfer-appropriate multifactor account, both of which focus on the importance of the relationship between encoding and retrieval processes in the production of generation advantages.

In the procedural account (Crutcher \& Healy, 1989; McNamara \& Healy, 1995a, 1995b), it is assumed that when generating information at study, as opposed to reading it, learners are more likely to employ encoding procedures that can be reinstated in a later retention test. If these procedures are invoked on a later test, a generation advantage should occur; if not, a generation advantage should not occur.

In the transfer-appropriate multifactor account (deWinstanley et al., 1996) - built upon the two-factor account of Hirshman and Bjork (1988) and the multifactor account of McDaniel, Waddill, and Einstein (1988) - the act of generation is assumed to strengthen whatever type of information is actually used to perform the generation task. The consequence of the generation task for later memory performance thus depends on whether the information so enhanced is information to which a later test is sensitive. When there is a good match between these types of information, generation advantages should occur; when there is not, they should not occur.

Thus, in either account, both the occurrence of generation advantages and the lack thereof depend on the relationship between encoding and retrieval processes. Consequently, both frameworks are able to explain a large set of findings for which changes in a variety of factors - such as the type of test that learners expect; whether to-be-read or to-be-generated items are mixed together; and the specific requirements of the generation task - have led to a continuum of outcomes ranging from large to small to no generation advantages.

\section{Sensitivity to Generation As an Effective Condition of Learning}

Recently, deWinstanley and Bjork (2004) addressed the general question of whether learners-if made sensitive to the memorial benefits of generation - would then adopt more effective processing strategies in the acquisition of new information. Although previous research had shown that giving learners specific instructions on how to improve their encoding of to-be-read information before study could eliminate the occurrence of generation effects, this more recent research explored whether generation advantages could be eliminated without participants' being explicitly instructed in how to process the to-be-read information more effectively. In particular, would giving learners the opportunity to experience a recall advantage in their own test performance for items that they had generated versus read be sufficient to induce more effective processing of future to-be-read information? Or, put slightly differently, could such an experience lead learners to discover for themselves how to become better readers?

To answer this question, deWinstanley and Bjork (2004) adopted the following general research strategy: Participants were presented with a short passage of the type that would appear in an introductory psychology textbook containing both to-be-generated and to-be-read critical items. Next, the participants' recall for these critical items was assessed in a fill-in-the-blank test. Following this test, 
a new text passage, also containing both to-be-generated and to-be-read critical items, was presented for study and then followed by the same type of test. Thus-before presentation of the second text passage-participants had engaged in both generating and reading critical items in a previous passage and would have had the opportunity to experience a generation advantage in their own performance on the memory test of those items. If, as deWinstanley and Bjork reasoned, such an experience is sufficient to induce participants to adopt a more effective way of encoding future to-be-read information, a generation advantage should be attenuated, or possibly eliminated, in the test of the second passage.

Employing this procedure in their first experiment, deWinstanley and Bjork (2004) obtained results consistent with this hypothesis. Although a generation advantage was observed in the test of the first passage, no generation advantage was observed in the test of the second passage. Importantly, however, the absence of a generation advantage on the second test did not occur at the expense of the generated items; instead, recall of the to-be-read items presented in the second passage improved to the level of that for the to-be-generated items, which did not differ from their level of recall in the test of the first passage.

In a second experiment, deWinstanley and Bjork (2004) both replicated this pattern of results with new passages and sought to assess whether participants became consciously aware of the memorial advantage of generation during the test of the first passage, a type of awareness that would seem to depend on a fairly sophisticated form of source memory. Following completion of the first test, participants were asked this open-ended question: "What did you notice about your performance on the previous memory test?" If, as deWinstanley and Bjork (2004) speculated, the opportunity for participants to experience the generation advantage in their own memory performance leads them to develop more effective encoding strategies, then some of their responses might reflect this awareness. deWinstanley and Bjork (2004) coded any mention by participants of their thinking that they remembered words better when they had "to complete" them or had "to figure" them "out" as indicating an awareness of a recall advantage for generated items; accordingly, just over half of the participants revealed this awareness. Most of these participants then processed the to-be-read items in the second passage more effectively, as indicated by their improved recall of such items.

In two follow-up experiments, deWinstanley and Bjork (2004) attempted to test more directly that it was the opportunity to experience the memorial consequences resulting from generating versus reading in the same testing episode that was responsible for participants' developing more effective encoding strategies. In the first follow-up, they used the same basic procedure of presenting two passages, each followed by an immediate test, but-rather than present both to-be-generated and to-be-read items within the same passage - they manipulated the requirement to generate versus read between passages. Thus, in the first passage for any one participant, the encoding task for all critical items was the same-either generating or reading - and, then, in the second passage, the encoding task for critical items was switched. As a result, the participants did not have the opportunity to experience the memorial consequences of generating versus reading on the same test prior to being presented with a new passage for study. If such an experience is critical in leading learners to adopt more effective encoding strategies for future to-be-read items, a generation advantage should not be eliminated in the test of the second passage, and indeed this result was obtained. More specifically, a generation advantage was obtained on both tests, and furthermore, the size of this advantage did not differ across tests. Apparently, then, participants encoding critical items via generation in the first passage did not become aware of the need to develop a better processing strategy for to-be-read critical items in the second passage - a result consistent with the importance of experiencing the relative memorial consequences of the two types of encoding within the same testing episode.

In the second follow-up, deWinstanley and Bjork (2004) examined whether something less specific_-like a general dissatisfaction with the number of read items that could be recalled in the first test - might have led participants to process future to-be-read items more effectively. This possibility was not ruled out by the first follow-up, because the switch of encoding tasks between passages made it impossible for participants presented with only to-be-read items in the first passage to reveal such improved encoding strategies for subsequent to-be-read items; only to-be-generated items were presented in the second passage. Thus, in the second follow-up, the requirement to generate versus read was manipulated between participants rather than between passages.

Although a generation advantage would be expected on the test of the first passage regardless of which hypothesis was correct, different outcomes would be expected on the test of the second passage. If something like a general feeling of dissatisfaction with one's ability to recall the to-be-read items is sufficient to induce a more effective processing strategy for future to-be-read items, then the generation advantage should be reduced or eliminated in the test of the second passage. If, however, the opportunity to experience the memorial benefits of generating relative to reading is critical for inducing such a processing change, then participants reading only critical items in the first passage should not change their processing strategy for the second passage, and a generation advantage should be seen on both tests. Consistent with the second hypothesis, a generation advantage was obtained in the tests for both passages and, as in the first follow-up, the size of this advantage did not differ across tests. Thus, when participants were denied the opportunity to experience the memorial advantage of generation in their own performance-either because the read-versus-generate encoding variable was manipulated between passages or because it was manipulated between participants - their ability to recall to-be-read items remained significantly poorer than their ability to recall to-be-generated items. 


\section{Remaining Questions and Potential Applications}

The research of deWinstanley and Bjork (2004) explored whether learners could discover for themselves how to become more effective readers. The pattern of results observed across four experiments indicated that experiencing the advantages of generation could induce learners to develop more effective encoding strategies. Or, in the terms of Koriat (1997), making learners sensitive to the power of generation as a learning event led them, in turn, to adopt enhanced strategies for the encoding of new information via reading.

These findings raise many interesting questions - some regarding the underlying cause of the effect observed by deWinstanley and Bjork (2004), and some regarding how these findings might best be applied to educational practices. Certainly a critical question concerns the effect's durability. In the experiments of deWinstanley and Bjork (2004), the second passage was always presented with little or no delay after the test of the first passage, raising the question of whether the testing experience leads to enhanced encoding of new information only when that information is presented immediately after the test. Perhaps, for example, a delay between the testing experience and the presentation of the next passage would prevent participants from adopting a more effective processing strategy for subsequent to-be-read information. A related issue would be whether the test experience must occur immediately after presentation of the passage in which participants have both generated and read critical items. Or, as might be necessary in educational settings, could the test be delayed without eliminating the learners' ability to benefit from the test experience? In ongoing research, we are addressing both of these questions and -importantly for the application of the present effect for educational practices - are obtaining results indicating that the presumed effect of the testing experience persists across a delay filled with other activities and, furthermore, that the test does not need to be administered immediately after presentation of the first passage.

Also of interest is the question of the necessity for learners actually to experience the differential effectiveness of encoding via generation versus reading in the context of a memory test. We know that this experience is critical because, in the deWinstanley and Bjork (2004) study, only the participants who were given this experience went on to adopt a more effective processing strategy for future to-be-read items. Although this finding is consistent with previous research indicating that learners are typically unable to judge the efficacy of a given processing strategy during its execution and do not switch from a less to a more effective strategy without an opportunity to experience their relative effectiveness (see, e.g., Dunlosky \& Hertzog, 2000), it is not clear whether the relative effectiveness must be experienced in the context of a testing episode. Perhaps, for example, simply instructing learners regarding the differential effectiveness of the two types of encoding might be sufficient. A related question would be whether, for the participants who did not reveal such an awareness in the posttest question asked by deWinstanley and Bjork (2004), it would be possible to lead them to such an awareness by giving them multiple passages and tests, rather than just two. Obtaining a better understanding of the metamemory processes involved in participants' becoming aware of the differential memorial consequences of generating versus reading also seems a fruitful avenue of research, one that might point to ways in which learners can become more metacognitively sophisticated (Bjork, 1999) in the management of their own learning.

Perhaps of most theoretical interest is the question of how participants improved their processing of to-be-read items in the second passage. One possibility is that participants used contextual information provided by other words in the passage to help them complete or encode the to-be-generated items and then used this information again in the subsequent fill-in-the-blank test to aid their recall. Indeed, such a strategy - to use contextual information first to complete and then to recall the generated items - could underlie the generation advantages observed on the tests of the first passages, an explanation that would be consistent with both the procedural and the transfer-appropriate multifactor accounts discussed earlier. In short, it would be the match between information strengthened during the generation task and the information needed to perform well on the later test, or the ability to reinstate during test the cognitive procedures used during study, that would have led to the generation advantages.

If this explanation for the initial generation advantage is correct, perhaps participants - having noticed both their superior recall of generated items and their use of such contextual information in recalling them - then attended to such contextual information during the study of the second passage for both types of critical items, thereby eliminating a generation advantage in their subsequent recall. This explanation would also be consistent with the finding that the generation advantage was not eliminated in tests of the second passage when participants had generated critical items only during study of the first passage. For such participants - although potentially using contextual information in the same way-the role of this strategy in aiding their recall may have been less apparent because they were recalling only items that they had generated and, thus, were not able to experience a contrast between their ability to recall words encoded via generation versus reading. Consequently, they would have been less likely to transfer the use of this strategy when encoding to-be-read items presented in the second passage. In research underway, we are attempting to test this notion by using different types of retention tests following study of the first passage - namely, ones that provide contextual information during the testing process and ones that do not. If, when tests do not provide contextual information, the testing experience does not lead to an elimination of a generation advantage for subsequently presented material, then the importance of such a strategy would be indicated.

Whatever is ultimately discovered as underlying participants' improved processing of to-be-read items in the deWinstanley and Bjork (2004) study, the basic findings seem to paint a promising picture from an applied 
perspective. First, deWinstanley and Bjork have clearly demonstrated that generation advantages do extend to the learning of the types of materials typically studied in textbooks. Furthermore, although how best to incorporate generation strategies into educational materials remains to be determined, one important implication of this research is that students will most likely need to experience for themselves the advantages of generation in the context of an actual test if they are to realize its effectiveness as a learning strategy. Thus, study exercises that just require generation are not likely to induce students into adopting more effective strategies when they read subsequently presented materials. More generally, it may prove necessary for students to experience the performance consequences of any differentially effective encoding processes before they can appreciate such differences and go on to make use of such knowledge in their future learning and study activities.

\section{AUTHOR NOTE}

Preparation of this report and some of the research reported therein was supported by Grant 29192G from the James S. McDonnell Foundation. Portions of this research were presented at the 2005 Annual Meeting of the Psychonomic Society, in Toronto. Correspondence should be addressed to E. L. Bjork, Department of Psychology, 1285 Franz Hall, University of California, Los Angeles, CA 90095 (e-mail: elbjork@ psych.ucla.edu).

\section{REFERENCES}

BegG, I., Vinski, L., Frankovich, L., \& Holgate, B. (1991). Generating makes words memorable, but so does effective reading. Memory \& Cognition, 19, 487-497.

Benjamin, A. S., Bjork, R. A., \& Schwartz, B. L. (1997). The mismeasure of memory: When retrieval fluency is misleading as a metamnemonic index. Journal of Experimental Psychology: General, 127, 55-68.

BJoRK, R. A. (1999). Assessing our own competence: Heuristics and illusions. In D. Gopher \& A. Koriat (Eds.), Attention and performance XVII: Cognitive regulation of performance. Interaction of theory and application (pp. 435-459). Cambridge, MA: MIT Press.

Crutcher, R. J., \& Healy, A. F. (1989). Cognitive operations and the generation effect. Journal of Experimental Psychology: Learning, Memory, \& Cognition, 15, 669-675.

DEWinstanley, P. A. (1995). A generation effect can be found during naturalistic learning. Psychonomic Bulletin \& Review, 2, 538-541.

DeWinstanley, P. A., \& Bjork, E. L. (1997). Processing instructions and the generation effect: A test of the multifactor transfer-appropriate processing theory. Memory, 5, 401-421.
DeWinstanley, P. A., \& Bjork, E. L. (2004). Processing strategies and the generation effect: Implications for making a better reader. Memory \& Cognition, 32, 945-955.

DeWinstanley, P. A., Bjork, E. L., \& Bjork, R. A. (1996). Generation effects and the lack thereof: The role of transfer appropriate processing. Memory, 4, 31-48.

Dunlosky, J., \& Hertzog, C. (2000). Updating knowledge about encoding strategies: A componential analysis of learning about strategy effectiveness from task experience. Psychology \& Aging, 15, 462-474.

Dunlosky, J., \& Nelson, T. O. (1994). Does the sensitivity of judgments of learning (JOLs) to the effects of various study activities depend on when the JOLs occur? Journal of Memory \& Language, 33, 545-565.

Hirshman, E., \& BJoRK, R. A. (1988). The generation effect: Support for a two-factor theory. Journal of Experimental Psychology: Learning, Memory, \& Cognition, 14, 484-494.

JACOBY, L. L. (1978). On interpreting the effects of repetition: Solving a problem versus remembering a solution. Journal of Verbal Learning \& Verbal Behavior, 17, 649-667.

JaCOBy, L. L., BJork, R. A., \& Kelley, C. M. (1994). Illusions of comprehension, competence, and remembering. In D. Druckman \& R. A. Bjork (Eds.), Learning, remembering, believing: Enhancing human performance (pp. 57-81). Washington, DC: National Academy Press.

Koriat, A. (1997). Monitoring one's own knowledge during study: A cue-utilization approach to judgments of learning. Journal of Experimental Psychology: General, 127, 349-370.

Koriat, A. (1998). Illusions of knowing: A window to the link between knowledge and metaknowledge. In V. Y. Yzerbyt, G. Lories, \& B. Dardenne (Eds.), Metacognition: Cognitive and social dimensions (pp. 16-34). London: Sage.

McDaniel, M. A., Waddill, P. J., \& Einstein, P. J. (1988). A contextual account of the generation effect: A three-factor theory. Journal of Memory \& Language, 27, 521-536.

McNamara, D. S., \& Healy, A. F. (1995a). A generation advantage for multiplication skill training and nonword vocabulary acquisition. In A. F. Healy \& L. E. Bourne, Jr. (Eds.), Learning and memory of knowledge and skills: Durability and specificity (pp. 132-169). Thousand Oaks, CA: Sage.

McNamara, D. S., \& Healy, A. F. (1995b). A procedural explanation of the generation effect: The use of an operand retrieval strategy for multiplication and addition problems. Journal of Memory \& Language, 34, 399-416.

Pesta, B. J., SANDERs, R. E., \& Murphy, M. D. (1999). A beautiful day in the neighborhood: What factors determine the generation effect for simple multiplication problems? Memory \& Cognition, 27, 106-115.

Simon, D., \& BJoRK, R. A. (2001). Metacognition in motor learning. Journal of Experimental Psychology: Learning, Memory, \& Cognition, 27, 907-912

Slamecka, N. J., \& Graf, P. (1978). The generation effect: Delineation of a phenomenon. Journal of Experimental Psychology: Human Learning \& Memory, 4, 592-604. 